\title{
The correlation between ion production and emission intensity in the laser-induced breakdown spectroscopy of liquid droplets
}

\author{
Jer-Shing Huang, Ching-Bin Ke, Li-Shing Huang, King-Chuen Lin* \\ Department of Chemistry, National Taiwan University, and Institute of Atomic and Molecular Sciences, Academia Sinica, \\ Taipei 106, Taiwan, ROC
}

Received 18 June 2001; accepted 4 September 2001

\begin{abstract}
Laser-induced breakdown spectroscopy (LIBS) is applied to detect trace metals contained in liquids. The sample in the form of liquid droplets is generated with an electrospray ionization needle. The microdroplets are interacted with an impinging laser pulse approximately $2 \mathrm{~mm}$ downstream from the needle tip. A sequence of single-shot time-resolved LIB emission signals of $\mathrm{Na}, \mathrm{K}$ and $\mathrm{Al}$ is detected, respectively. The LIB signal intensity integrated within a gate linearly correlates with the plasma current obtained simultaneously on a single-shot basis. The correlation plot exhibits a straight line, of which the slope increases with the sample concentration. Given the calibration curves and the focused cross-sections of the incident laser beam, the detection limits may be determined to be $0.63 \pm 0.02(0.3 \mathrm{pg}), 1.2 \pm 0.1(0.5 \mathrm{pg})$, and $43 \pm 5 \mathrm{mg} / \mathrm{l}(21 \mathrm{pg})$ for $\mathrm{Na}, \mathrm{K}$, and $\mathrm{Al}$, respectively. As compared to the correlation methods applied, our treatment is more straightforward to yield concentration information and the resulting detection limits are comparable to those reported previously. The dependence of the correlation plot on the adopted laser energy and wavelength is also characterized. The detection limits tend to be improved by applying a laser with larger pulse energy but shorter wavelengths. (c) 2002 Elsevier Science B.V. All rights reserved.
\end{abstract}

Keywords: Laser-induced breakdown spectroscopy; Electrospray ionization; Correlation method; Elemental analysis

\footnotetext{
* Corresponding author. Fax: +886-2-23621483.
}

E-mail address: kclin@ccms.ntu.edu.tw (K. Lin). 


\section{Introduction}

Laser-induced breakdown spectroscopy (LIBS) is a potential technique for rapid analysis of environmental samples present in solids, gases, and liquids [1-10]. The merits of high sensitivity, noninvasive analysis, suitability to remote measurement, and minimal sample preparation have made this technique become increasingly attractive.

Various approaches of LIBS have been developed to monitor metal elements contained in liquid solutions [7,9,11-17]. Aside from the common interest in the limit of detection (LOD), factors affecting analytical sensitivity are often investigated too, such as dependence of pulse energy and wavelength [7,17], comparison of surface, bulk, or droplet excitation [7,11,13,14], and application of dual-laser techniques [16]. Bulk liquid and liquid jet are commonly used as sampling methods to minimize the signal fluctuation. But the detection limits for most metal elements stay in the range of $\mathrm{mg} / \mathrm{l}(\mathrm{ppm})$. The poor signal quality is partly caused by the laser-pulse fluctuation, inconsistency of the sampled amount of medium, and instability of the plasma position. It is also attributed to bremsstrahlung radiation under which the discrete emission lines of the sample may be buried in a strong continuum background [3-5].

One drawback of the laser-induced breakdown (LIB) technique lies in the fact that the LOD for a liquid analyte is substantially reduced by large shot-to-shot signal variation. The conventional method to overcome the fluctuation problem is by averaging the spectral signals over a number of laser shots. Nevertheless, the improvement of the signal-to-noise $(\mathrm{S} / \mathrm{N})$ is offset by the non-linear optical ablation effect and the enhanced $\mathrm{S} / \mathrm{N}$ ratio may fail to reach the prediction by $N^{1 / 2}(N$ is the number of laser shots) [7,9]. The internal standard method is another popular solution via the measurement of the intensity ratio of the analyte and the reference element to compensate for the signal fluctuation. The reference element is assumed to have a constant concentration and the same fluctuation pattern as that of the analyzed sample [6,18-20].

The treatment in terms of LIB emission nor- malization has been similarly applied elsewhere. For instance, Cheung and Young [7] have demonstrated single-shot elemental analysis in the liquid detection based on pulsed laser vaporization at sub-breakdown fluences, together with the acoustic normalization for improved precision. The intensity of normalized LIB signal shows a linear proportion to the laser pulse energy. The resulting limits of detection (LOD) of $\mathrm{Na}, \mathrm{K}$ and $\mathrm{Ba}$ reach $0.23,1.5$ and $130 \mathrm{ppm}$, respectively. In detecting trace metals in soil and aerosols, Schechter and co-workers [9] have measured a sequence of spectra from single breakdown events. The line peak of the analyte is found to correlate well with the baseline, which has the same multiplicative fluctuation as the sample signal. The correlation plot of analyte signal vs. background may determine the sample concentration. This method, as it is pointed out, does not depend on a constant constituent of a reference element involved $[6,18,19]$.

The electrospray ionization technique has been popularly used as a desorption ion source in molecular mass spectrometry for analyzing nonvolatile biomolecules with large molecular weight. A solution of the sample is pumped through a high voltage-biased capillary needle. The resulting charged spray of tiny droplets then passes the desolvating capillary, where evaporation of solvent and attachment of charge to the analyte take place. In this work, the electrospray ionization technique is employed for the first time to generate microdroplets for the elemental analysis by LIBS. The generation of microdroplets may facilitate sample vaporization, atomization and excitation/ionization efficiency. In addition, as the microdroplets are ionized by LIBS, the current induced by the high voltage biased on the ionization needle may be feasibly collected. In the LIB emission of the analyte, we observed the characteristics correlating with the corresponding current, which lead the plot of LIB emission together with the current normalization to a great reduction of the signal fluctuation.

By the electrospray ionization technique, we successfully demonstrate in this work the singleshot LIB analysis for $\mathrm{Na}, \mathrm{K}$ and $\mathrm{Al}$ elements. Given constant laser energy, we find that the 
intensities of single-shot time-resolved LIB emission linearly correlates with the currents collected from the corresponding pulse-induced plasma. The correlation plot yields a slope, which increases in proportion to the sample concentration. The calibration curves of the concentrations and the subsequent limits of detection (LOD) for $\mathrm{Na}, \mathrm{K}$ and $\mathrm{Al}$ are determined. The correlation method is also characterized by studies of laser pulse energy and wavelength dependence.

\section{Experimental setup}

A schematic diagram for the LIBS system is displayed in Fig. 1. It contains three parts: (1) laser source; (2) detection system; and (3) electrospray assembly.

\subsection{Laser source}

An Nd:YAG laser (GCR-3, Spectra-Physics)

(a)

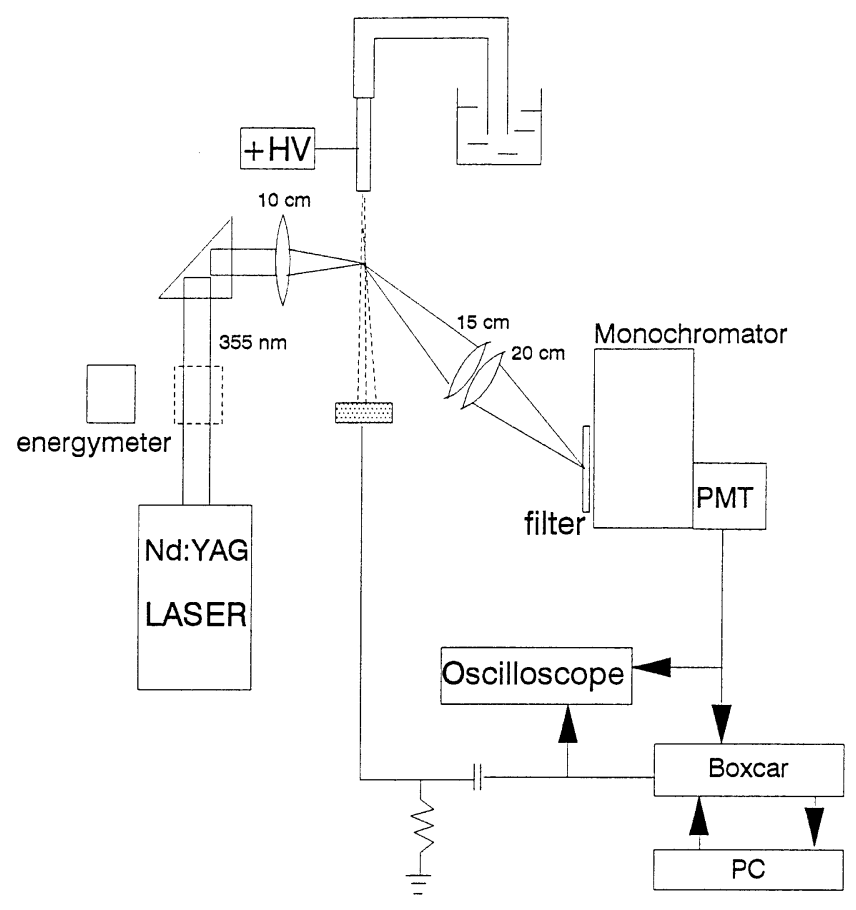

(b)

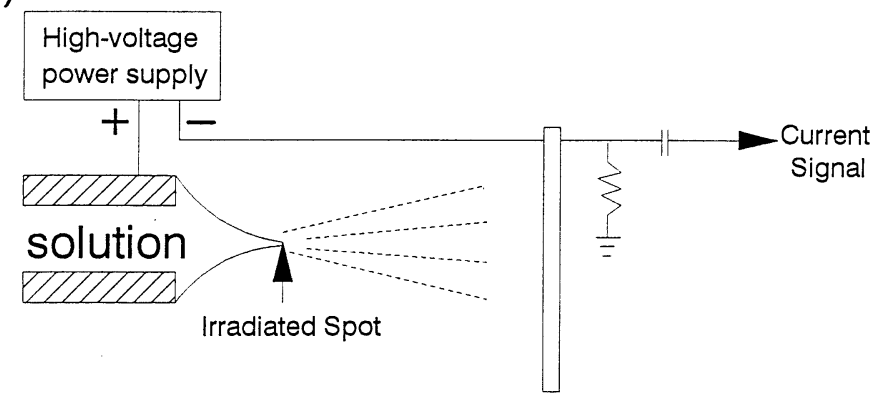

Fig. 1. (a) Schematic diagram for the setup of laser-induced breakdown spectroscopy. (b) Design of the current collector. The irradiated spot by laser pulse indicated is close to the Taylor cone generated by the electrospray ionization needle. 
performed at the second, third, and fourth harmonic generation emitting at 532, 355, and 266 $\mathrm{nm}$, respectively, was used as the breakdown wavelengths. The laser, Q-switched through a Pockel cell, was operated at $10 \mathrm{~Hz}$ with a pulse duration of 5-8 ns. The incident pulse was focused through a $10-\mathrm{cm}$ focal length quartz lens onto the microdroplets generated by an electrospray ionization needle. The laser pulse energy prior to the quartz lens was controlled in the range from 10 to $45 \mathrm{~mJ}$. The pulse-to-pulse fluctuation was expected to be $\pm 5 \%$.

\subsection{Detection system}

Upon laser-pulse interaction with the liquid droplets, the produced luminous plume was imaged with a set of $15-$ and $20-\mathrm{cm}$ focal length lenses onto a 0.35 -m Czerney-Turner monochromator (Model 270, McPherson) behind which a photomultiplier tube (PMT, R955, Hamamatsu) was mounted. The LIBS emission was collected in perpendicular direction relative to the impinging laser beam. The monochromator equipped with a 1200 -groove $/ \mathrm{mm}$ grating possessed a reciprocal linear dispersion of $2 \mathrm{~nm} / \mathrm{mm}$. The grating was centered at 589, 766 and $396 \mathrm{~nm}$ for the detection of the $\mathrm{Na}, \mathrm{K}$ and $\mathrm{Al}$ atoms in the emission transitions of $3^{2} \mathrm{P} \rightarrow 3^{2} \mathrm{~S}, 4^{2} \mathrm{P} \rightarrow 4^{2} \mathrm{~S}$ and $4^{2} \mathrm{~S} \rightarrow 3^{2} \mathrm{P}$, respectively. The slits were open to $500 \mu \mathrm{m}$ in width, to transmit the total plasma plume which was magnified by a factor of 1.3 as imaged onto the entrance slit. The use of the monochromator has two advantages. First, its grating can be feasibly tuned to a particular wavelength for the elements studied and thus most spectral interference may be effectively reduced. Second, the width of the slits could be adjusted to reduce the transmitted continuum background emission at this particular wavelength. Thus the PMT detector needs no protection of a shutter and can be exposed safely below optical saturation.

The obtained time-resolved LIB emission was fed into either a transient digitizer (Model 9450A, LeCroy) for single-shot profile recording or a boxcar integrator (Model SR250, Stanford Research System) for signal processing. For eliminating the interference of the continuum back- ground emission, the time-resolved spectra were integrated within a gate on a single-shot basis. The gate width and position over the time-resolved spectra must be optimized to achieve a linear correlation between the emission intensity and the plasma-related current obtained simultaneously. In this work, the gate width optimized for the $\mathrm{Na}$ analysis was $14 \mu \mathrm{s}$; the delay time was 900 ns relative to the trigger of the laser pulse. The same conditions were also valid for the $\mathrm{K}$ and $\mathrm{Al}$ analyses.

\subsection{Electrospray}

A homemade electrospray ionization device was employed to generate a stream of microdroplets. The analyte solution was supplied by a PTFE tube, which was connected to a $200-\mu \mathrm{m}$ i.d. stainless-steel spray needle. The needle served as the anode, biased at $3 \mathrm{kV}$, through which the analyte solution was sprayed toward the other metal base used as the cathode. The bias voltage was supplied by a Glassman EH-series high-voltage supply $(0-10 \mathrm{kV})$. As carrier solvent evaporated, the electrostatic charge density on the surface of the sprayed droplets increased. When the surface charge density increased to a critical point, the resultant electrostatic repulsion broke the droplets into smaller ones [21,22]. This process was repeated to form a mist of very fine droplets. Their size and density decreased as the distance to the needle tip increased. The laser pulse interacted with the sprayed droplets at $\sim 2 \mathrm{~mm}$ downstream from the needle tip. The irradiated spot was close to the tip of Taylor cone to have a higher density of microdroplets.

\subsection{Current collection}

The current resulting from laser-induced plasma was collected from the cathode via a high-pass filter to enter a second channel of either a transient digitizer or a boxcar integrator. The current profile oscillated rapidly in a nanosecond scale, as displayed on the transient digitizer. Its maximum peak was then integrated within a 20 -ns gate provided by the boxcar integrator. The current was monitored simultaneously 
with the LIB emission on a single-shot event up to 200 pulses.

\subsection{Sample preparation}

The $\mathrm{Na}, \mathrm{K}$ and $\mathrm{Al}$ samples were made from their chloride solutions. A $1000-\mathrm{mg} / 1 \mathrm{NaCl}, \mathrm{KCl}$, and $\mathrm{AlCl}_{3}$ (Reagent grade, Merck) aqueous solutions were first prepared and then diluted to the desired concentrations by appropriate volume of methanol (HPLC grade, Baker). For gaining a better electrospray efficiency, we used a water/methanol $(\mathrm{v} / \mathrm{v}=1: 1)$ mixed solvent throughout this work. Since water and methanol had different densities, the concentration was indicated by the unit of $\mathrm{mg} / \mathrm{l}$ rather than $\mathrm{ppm}$.

\section{Results and discussion}

\subsection{LIB signal vs. current background}

\subsubsection{Single-shot LIB emission}

Fig. 2a shows an example of single-shot timedependence of the LIB emission intensity of $\mathrm{Na}$ detected from the sample microdroplets sprayed by the electrospray tip. The spectrum is integrated within a $14-\mu$ s gate in a 900-ns delay relative to the laser pulse. A sequence of the corresponding intensity of the LIB emission is given in Fig. 2b. The severity of the shot-to-shot signal variation in Fig. $2 \mathrm{~b}$ obviously hampers the improvement of the $\mathrm{S} / \mathrm{N}$ ratio of the spectral intensity averaged over 200 pulses. As shown in Fig. 3, $50 \mathrm{mg} / 1$ is the approximate upper limit of the linear dynamic range for the average LIB emissions of $\mathrm{Na}$ at different concentrations. Each data point carries a large error bar, which represents one standard deviation for the average over 200 laser pulses. The poor quality of the detection may be attributed to the following factors. First, the microdroplets change positions with time. As a result, the total amount interacted by the laser pulses varies from shot to shot. Second, the microdroplet interaction by the laser pulse is exposed to the air, and the laser-induced air breakdown in turn contributes to the background emission. Third, output energies of the laser source vary from pulse to pulse. Furthermore, the shapes and densities of the droplets generated by the electrospray tip change with the salt concentration. Increase of the salt concentration may increase the ion solvation strength, and thereby the electrospray efficiency is reduced [21,22]. This might be an important factor for the deviation of the calibration curve at $>50 \mathrm{mg} / 1$ in Fig. 3 from a linearity. Another important factor is selfabsorption of the atomic emission at high atom number density.

\subsubsection{Correlation between LIB and current}

Fig. 4a gives an example for the single-shot oscillating waveforms of the plasma-related current, which makes a clear distinction of the breakdown between air and a 100-mg/l Na solution. The corresponding current intensities are obtained from integration of the maximum peak within a constant gate. Fig. $4 \mathrm{~b}$ shows a sequence of such current intensities on single-shot basis from the laser-induced breakdown of the Na-sample droplets.

As shown in Fig. 4a, the oscillating character of the current signal, similar to that of the acoustic signal reported [7], might be caused by the electronic circuit involved. It might not be caused by the RF pickup from the laser flashlamp, since the rapid oscillation of the air disappears when the laser energy is reduced to the threshold of the air breakdown. But its physical significance is yet to be known. The current oscillation from the air breakdown contributes to the magnitude of background. Since the slope of the correlation plot is concerned, there is no need for background contribution to be subtracted from the signal measurements. Like the behavior of LIB emission, the current signal in Fig. 4b fluctuates seriously. However, each current shot appears to correlate well with the LIB emission response. As shown in Fig. 5, the plot of Na LIB emission intensity against the current intensity yields a straight line, of which the slope depends on the concentrations of the analyte solution. The slope increases as the concentrations increase. The obtained regression coefficients of the correlation plots, each derived from 200 data points, are larger than 0.95 . The upper limit in the linear dynamic range of the 

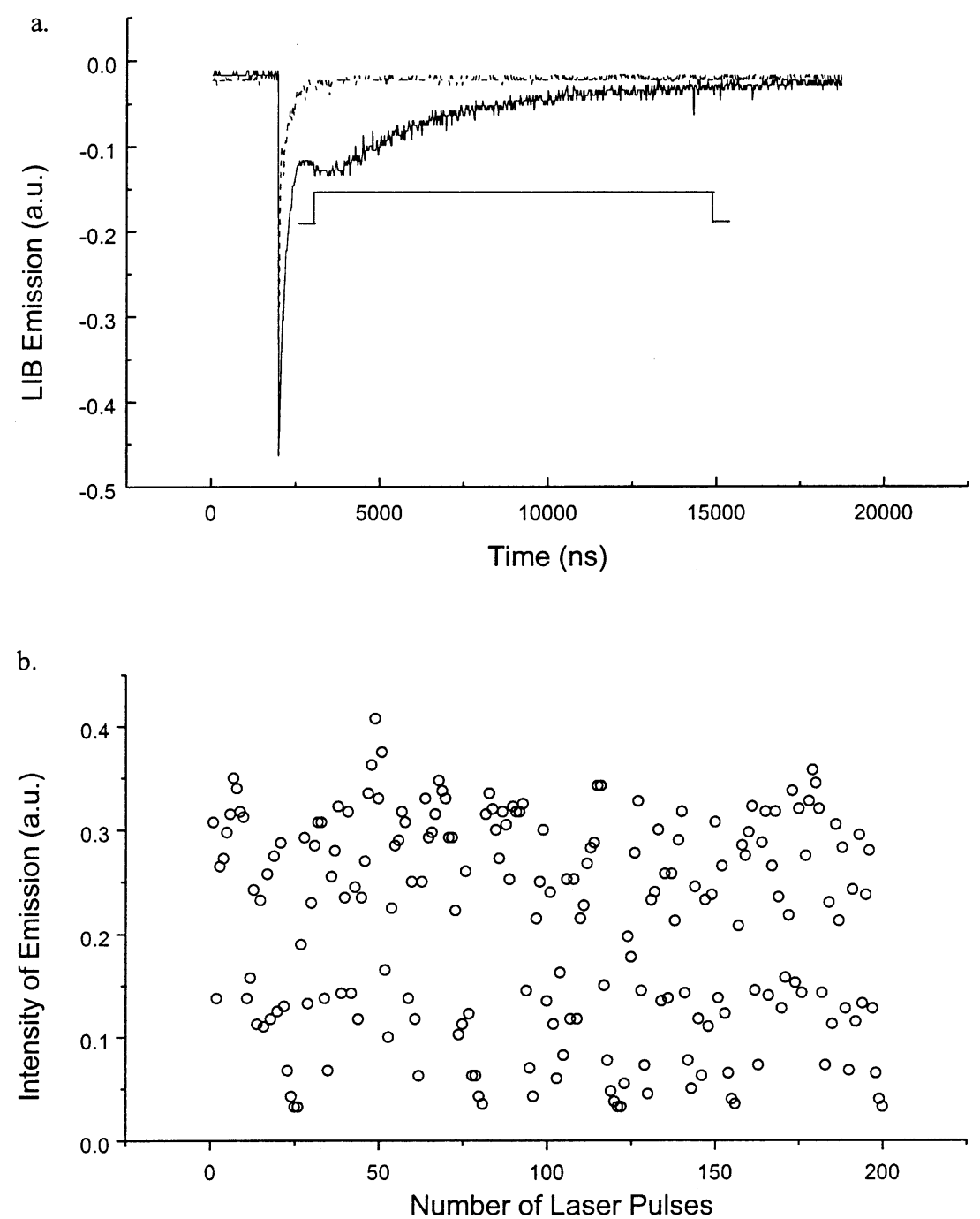

Fig. 2. (a) Single-shot time dependence of LIB spectra for a $100-\mathrm{mg} / 1 \mathrm{Na}$ solution (-) and air (---). The gate is set at $14 \mu \mathrm{s}$ in width and delayed by $900 \mathrm{~ns}$ relative to the trigger of the laser pulse. The spectrum within the gate is integrated to obtain the intensity of LIB emission. (b) Intensities of single-shot Na LIB emission for 200 data points. The laser pulse is at $355 \mathrm{~nm}$ with energy of approximately $13 \mathrm{~mJ}$.

calibration curves, represented by the plots of the obtained slopes vs. the corresponding sample concentrations, can be extended at least to 200, 250 and $900 \mathrm{mg} / \mathrm{l}$ for K, Na, and Al, respectively. Fig. $3 \mathrm{a}, \mathrm{b}$ gives a comparison for the calibration curves of $\mathrm{Na}$ with and without the treatment with the slopes of the current correlation plots. A much better precision found in Fig. 3b is apparently due to the data treatment with the current correla- tion. Fig. 6 shows another calibration curve of the $\mathrm{Al}$ case with the correlation treatment. As shown in Fig. 3b and Fig. 6, the calibration curves do not pass through the origin. It is caused by the fact that the slope of the correlation plot for the blank solution is not zero. The same magnitude of background is contributed to all other measurements for different concentrations of the sample. Therefore, the non-zero background does not affect the 

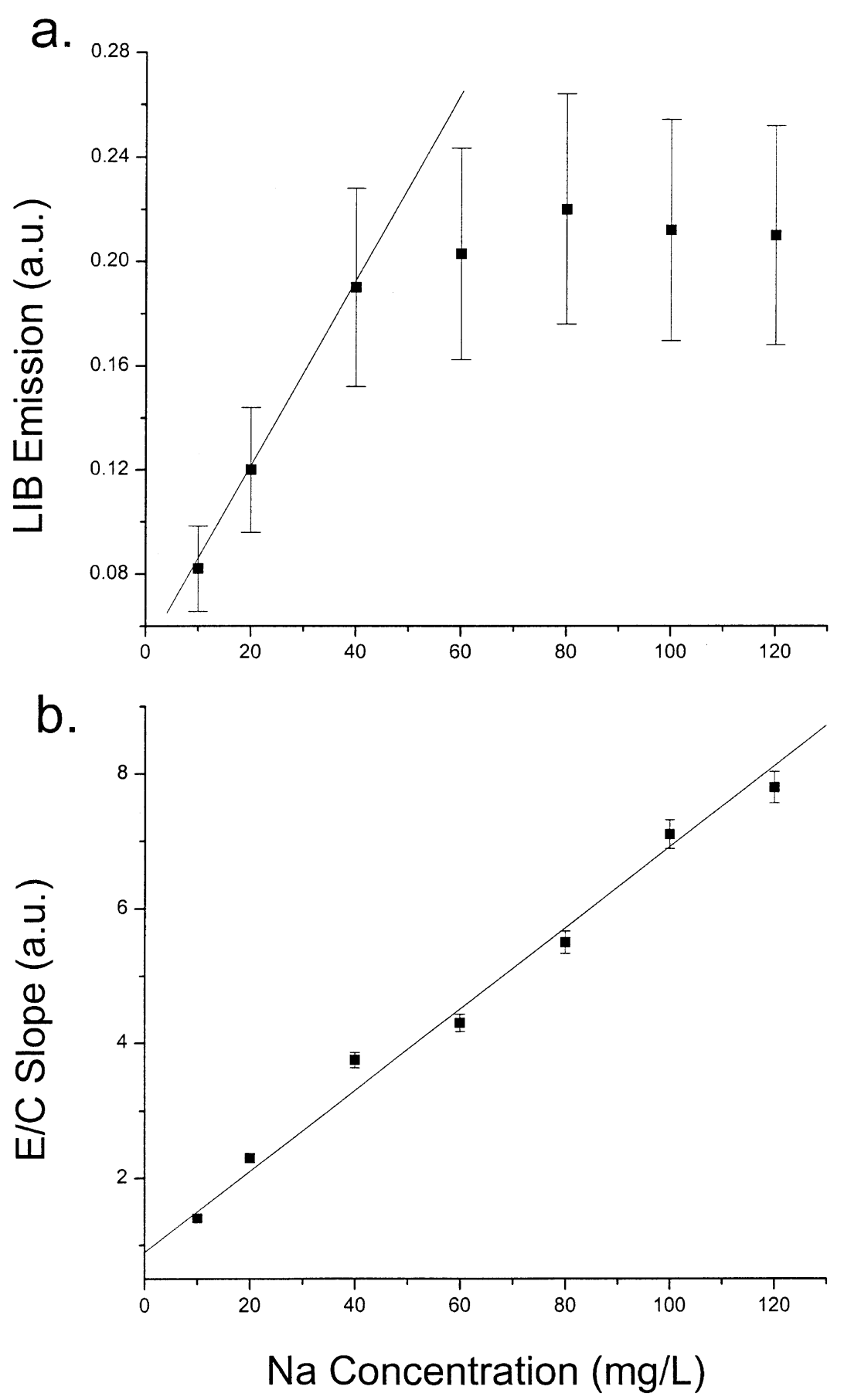

Fig. 3. (a) Calibration curve of $\mathrm{Na}$ at different concentrations without correlation treatment. Each data point of LIB emission intensity is averaged over 200 pulses with an error bar of 1 S.D. Laser pulse is at $355 \mathrm{~nm}$ with energy of approximately 13 mJ. (b) Calibration curve of $\mathrm{Na}$ at different concentrations with correlation treatment. The $\mathrm{E} / \mathrm{C}$ slope is obtained from the correlation plot of each single-shot LIB emission intensity in (a) vs. the corresponding current. 

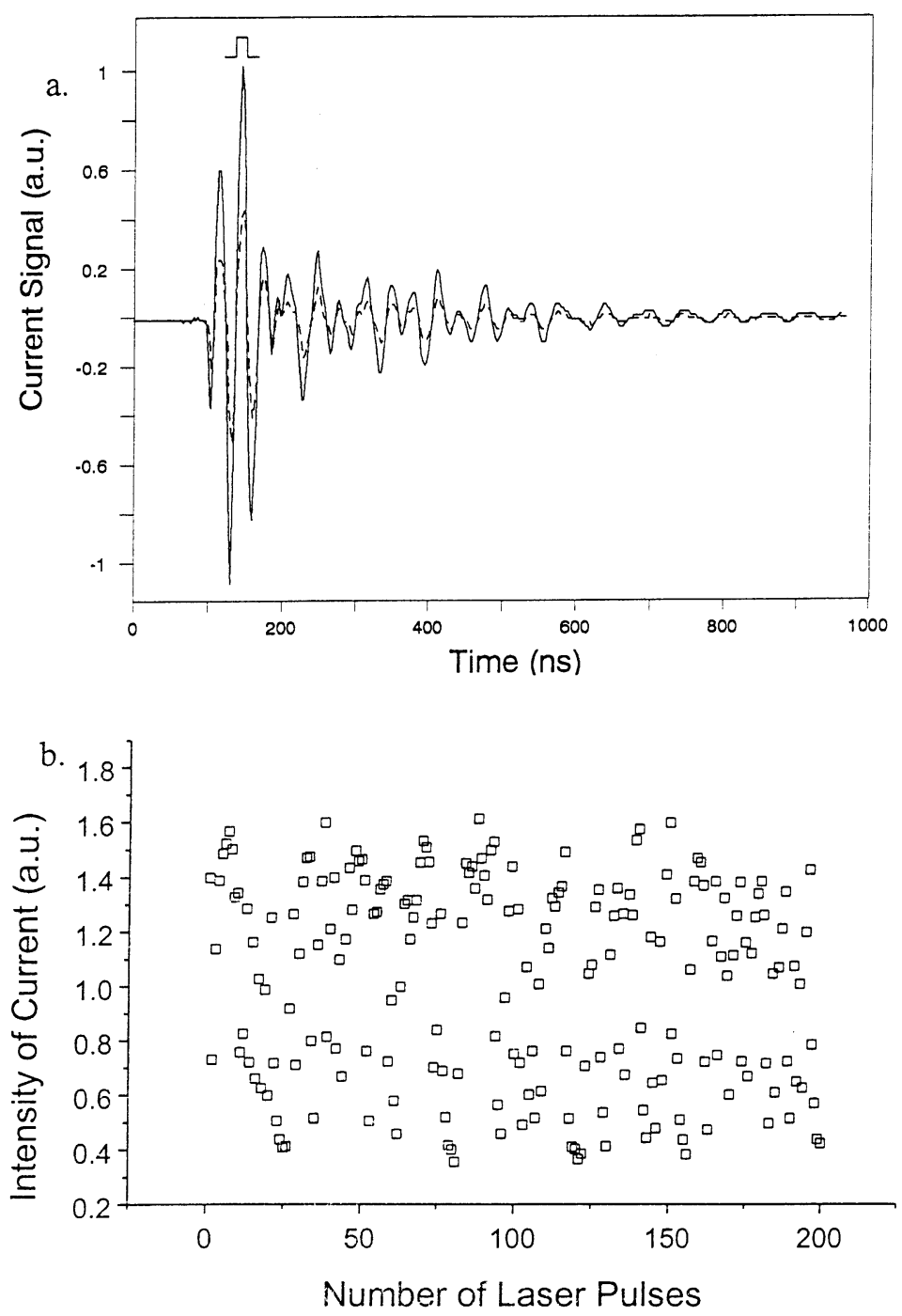

Fig. 4. (a) Current waveforms detected from a $100-\mathrm{mg} / 1 \mathrm{Na}$ solution (-) and air (---), respectively. The maximum peak is integrated within a gate to obtain the current intensity. (b) Single-shot current intensities collected from the laser-induced plasma for 200 data points. The laser pulse is at $355 \mathrm{~nm}$ with energy of approximately $13 \mathrm{~mJ}$.

determination of the sensitivity in the calibration curve.

The detection limit is defined as $2 \sigma / s$, in which $\sigma$ is the standard deviation of the blank measurement and $s$ is the slope of the calibration curve. The detection limits for the $\mathrm{Na}, \mathrm{K}$ and $\mathrm{Al}$ samples are accordingly estimated to be $0.63 \pm 0.02,1.2 \pm$ 0.1 , and $43 \pm 5 \mathrm{mg} / \mathrm{l}$, respectively. As listed on Table 1, the obtained detection limits are comparable to most results reported previously, al- though our measurements are under the conditions of plasma instability and thin microdroplet density. With regard to the conversion of absolute quantities detected, the size distribution and the number density of the microdroplets in the probe region should be taken into account. However, it is not trivial to gain these results. We assume that the droplet amounts interacted by the laser pulse remain constant like in a bulk solution. Then the conversion of absolute quantities needs only to 

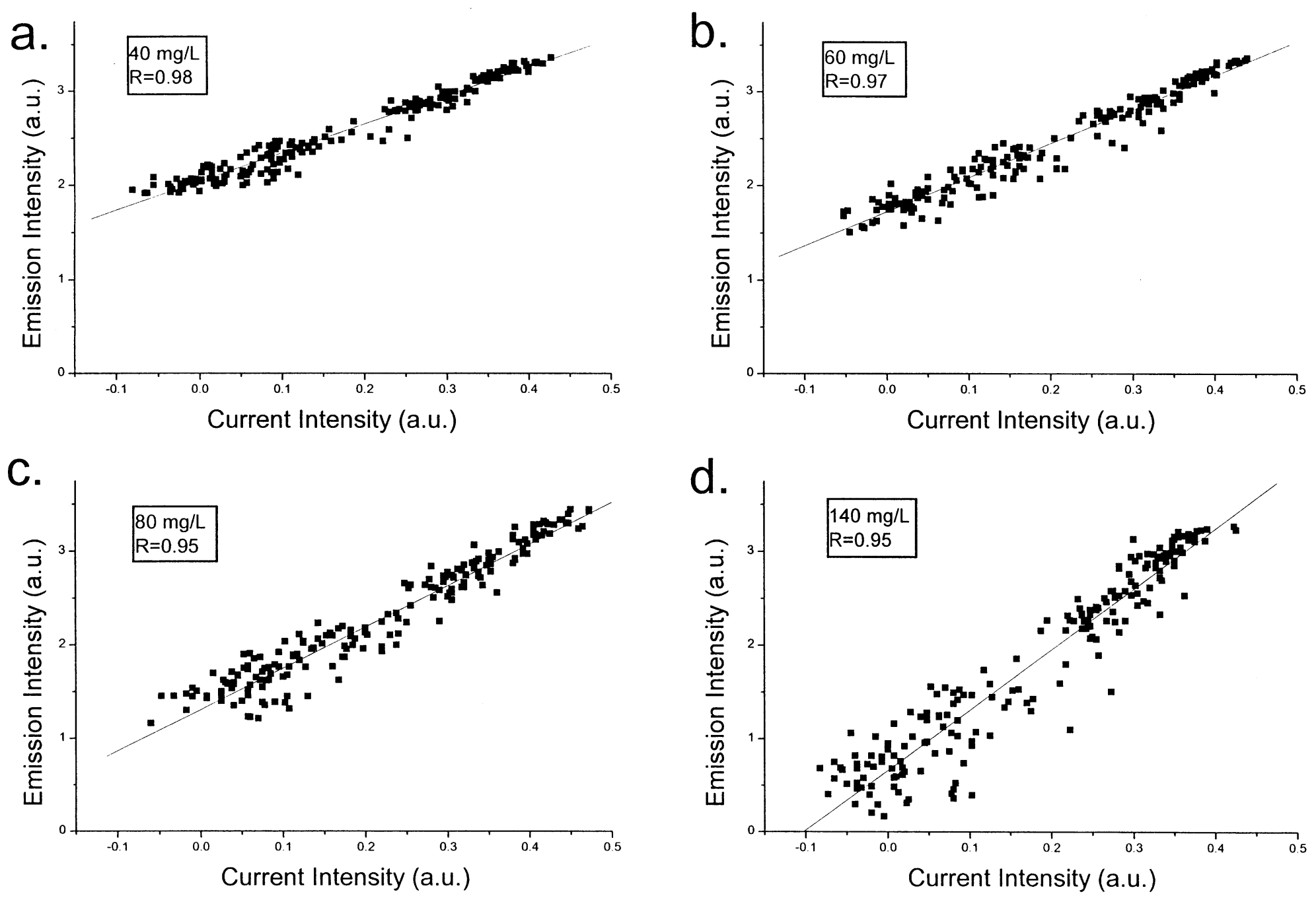

Fig. 5. (a) Correlation plot of the Na LIB emission signal vs. the current signal. (a) A 40-mg/1 Na solution, slope $=3.04$; (b) a 60 -mg $/ 1$ Na solution, slope $=3.62$; (c) a $80-\mathrm{mg} / 1 \mathrm{Na}$ solution, slope $=4.42$; and (d) a $140-\mathrm{mg} / 1 \mathrm{Na}$ solution, slope $=6.46$. The laser pulse is at $355 \mathrm{~nm}$ with energy of approximately $13 \mathrm{~mJ}$. 


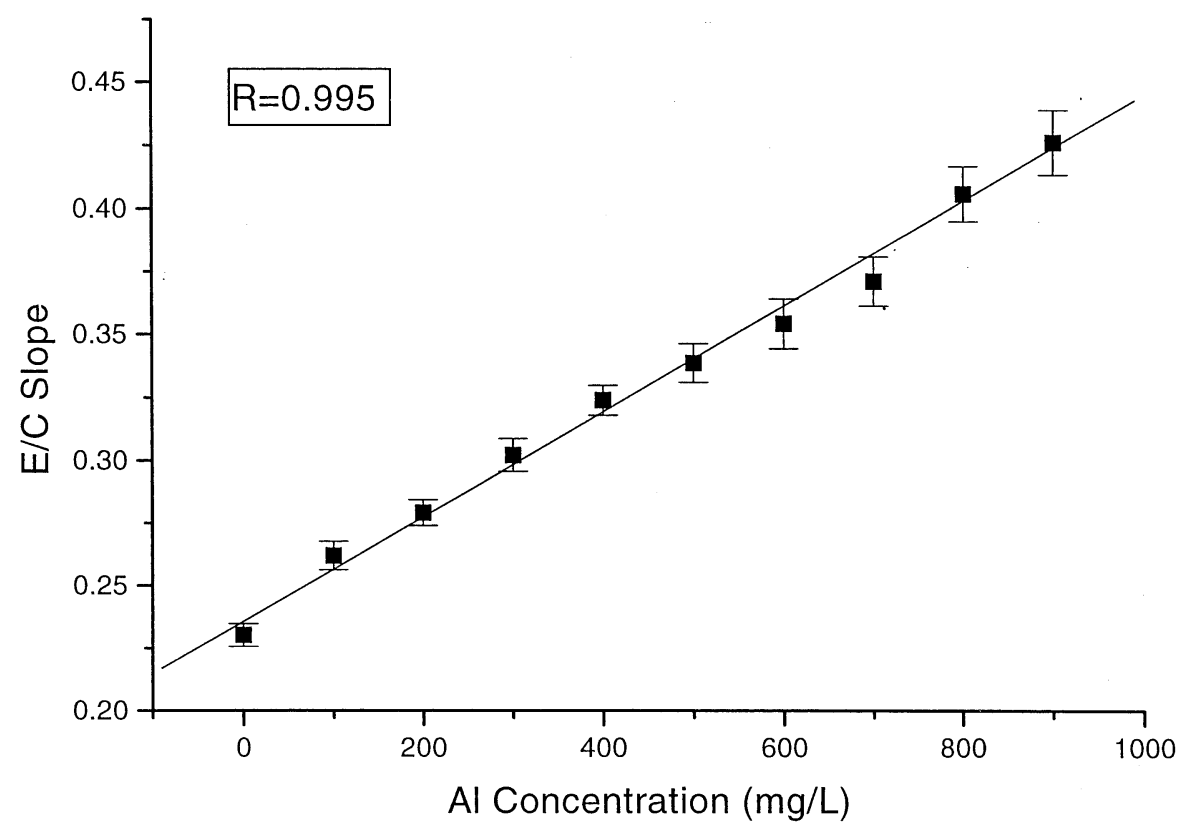

Fig. 6. Calibration curve of $\mathrm{Al}$ concentration. The $\mathrm{E} / \mathrm{C}$ slope, indicative of the LIB emission with current normalization, is obtained from the correlation plots at various concentrations, as given similarly in Fig. 5.

consider the interactive volume, which is determined by the beam waist of the focused spot. Accordingly, a magnitude of $0.3,0.5$ and $21 \mathrm{pg}$ for the $\mathrm{Na}, \mathrm{K}$ and $\mathrm{Al}$ elements may be evaluated in a single-pulse measurement, assuming the focused beam waist is $10^{-4} \mathrm{~m}$ [7].

\subsection{Characterization of current response and correlation plot}

\subsubsection{Current characterization}

The current induced by the plasma generation is composed of three sources. First, as the irradiated droplets are ionized sufficiently, the droplet plasma is formed from the ionization of water, methanol and sample salt. Second, the air ionization partly contributes to the background plasma. Third, the charged droplets, induced initially in the electric field prior to the laser irradiation, contribute to the direct current (DC) signal. However, this DC contribution may be eliminated by a high-pass filter installed in the current collector.

When the pulse energy is large enough to ionize the droplets, the current is collected substan- tially from the electrons, which move more rapidly than the ions and are in a much shorter distance toward one end of the collector [23,24]. The electrons result mainly from the ionized water/ methanol droplets and their fragments, while little from the contained sample salt. Such polyatomic molecules as the solvent may lead to the electron multiplication as fragmented and ionized. This makes the estimation of the efficiency with which the LIB technique ionizes the sample become highly complicated, although the total ion pairs per single laser pulse may be estimated by integrating the current waveform. Unlike the LIB technique, a laser-enhanced ionization spectrometer has been successfully applied to determine the total atom density of the sample and the subsequent atomization efficiency in flames $[25,26]$.

The current response induced by LIB simply reflects the droplet size and density, irrespective of the salt concentration contained. Considering that the total quantity of the sample salt depends on the droplet size and density, a strong/weak current intensity is accompanied by a strong/weak 
Table 1

Comparison of detection limits (mg/l) for $\mathrm{Na}, \mathrm{K}$ and $\mathrm{Al}$ in LIBS

\begin{tabular}{lllll}
\hline $\mathrm{Na}$ & $\mathrm{K}$ & $\mathrm{AI}$ & Method & Ref. \\
\hline $\begin{array}{l}0.6 \pm 0.1^{\mathrm{a}} \\
(0.3 \mathrm{pg})\end{array}$ & $\begin{array}{l}2.7 \pm 0.1^{\mathrm{a}} \\
(1.2 \mathrm{pg}) \\
1.2 \pm 0.1^{\mathrm{b}}\end{array}$ & $43 \pm 5^{\mathrm{c}}$ & Electrospray & This work \\
& $(0.5 \mathrm{pg})$ & $(21 \mathrm{pg})$ & & \\
0.23 & 1.5 & & Liquid jet & [7] \\
$(0.4 \mathrm{pg})$ & $(2.6 \mathrm{pg})$ & - & Bulk liquid & {$[11]$} \\
0.014 & 1.2 & & & Isolated droplet \\
$(14 \mathrm{pg})$ & $(1200 \mathrm{pg})$ & 20 & $\left(2 \times 10^{4} \mathrm{pg}\right)$ & {$[13]$} \\
2.2 & - & 5.2 & & \\
$\left(4.4 \times 10^{5} \mathrm{pg}\right)$ & & $\left(1 \times 10^{6} \mathrm{pg}\right)$ & & \\
\hline
\end{tabular}

${ }^{\mathrm{a}} \mathrm{A}$ 355-nm laser pulse used at $12.6 \mathrm{~mJ}$.

${ }^{\mathrm{b}} \mathrm{A}$ 266-nm laser pulse used at $12.6 \mathrm{~mJ}$.

${ }^{\mathrm{c}} \mathrm{A}$ 355-nm laser pulse used at $38 \mathrm{~mJ}$.

response of the LIB emission. In addition, increase of the sample concentration may increase the LIB emission intensity, but does not change the current response significantly. The slope in the correlation plot may therefore sensitively reflect the sample concentration.

\subsubsection{Comparison among correlation methods}

Our work is similar to the studies made by Young and co-workers [7], Schechter and coworkers [9], and Winefordner and co-workers [20] in the treatment of correlation analysis adopted, but differ in the related sampling processes and data treatments. First, when the irradiated spot area is assumed similar, in our work the detected sample amount from a mist of microdroplets is less than that with the liquid jet or bulk liquid. Nevertheless, the resulting detection limits are comparable to most results reported with the latter sampling method $[7,11,13]$. Second, the correlation slopes obtained at fixed pulse energy in this work can be used directly to set up a calibration curve without any algebraic model involved. In contrast, in the correlation treatment reported by Schechter and co-workers [9], the correlation slope has to subtract a unity for the removal of background emission before adoption in the calibration curve. Winefordner and co-workers [20] have recently examined applicability of the alge- braic model proposed in this method. Cheung and Young [7], by applying LIBS in a liquid jet, have found that the single-shot LIB signal with the corresponding acoustic normalization shows a linear relationship with the impinging pulse energy. The slope obtained by the plot of normalized LIB emission vs. pulse energy may yield information of the sample concentration. The correlated signals need to be treated differently, since the data points are clustered in a small region.

\subsection{Dependence of laser energy and wavelength}

The detection limits in the elemental analysis are affected by laser pulse energy and wavelength. As shown in Fig. 7, the slope in the calibration curve for the $\mathrm{K}$ solution increases as the pulse energy increases from 20 to $40 \mathrm{~mJ}$. The corresponding detection limits are therefore improved from 3.5 to $2.9 \mathrm{mg} / \mathrm{l}$. The pulse energy is increased twofold, but the detection limit is lowered by only $17 \%$. The little improvement may be caused by at least two factors. First, the population in the excited state is reduced as a result of competition of the ionization process, which is substantially enhanced with an increase of the laser energy. Thus the LIB emission intensity of $\mathrm{K}$ at $766 \mathrm{~nm}$ cannot be enhanced as expected. Second, the conditions adopted for integration of 
a.

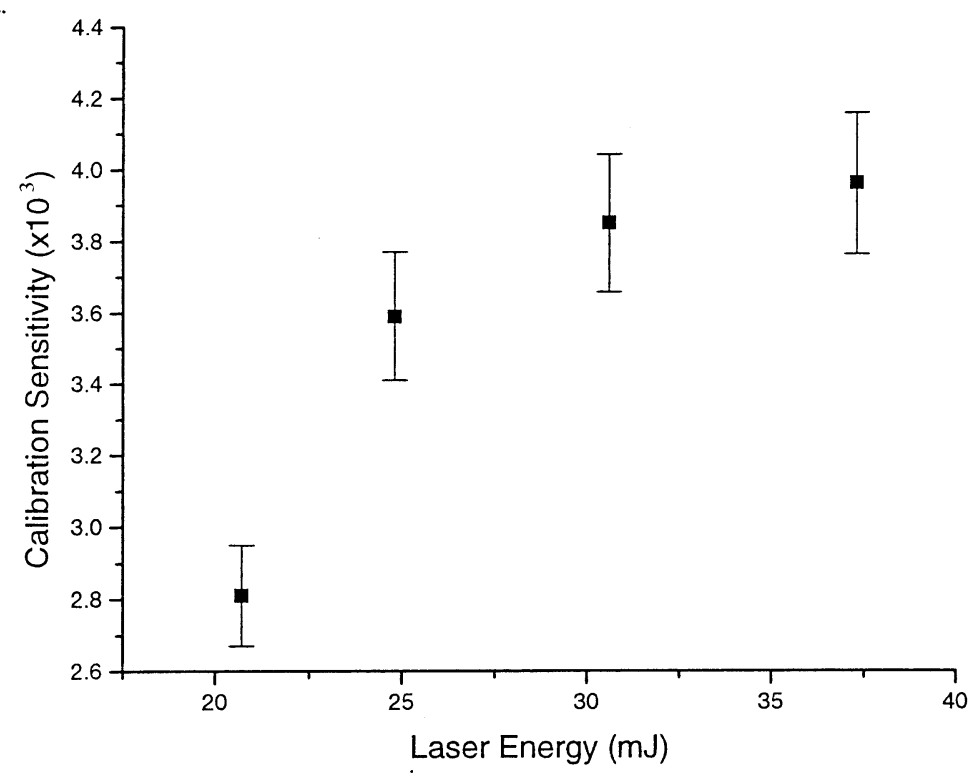

b.

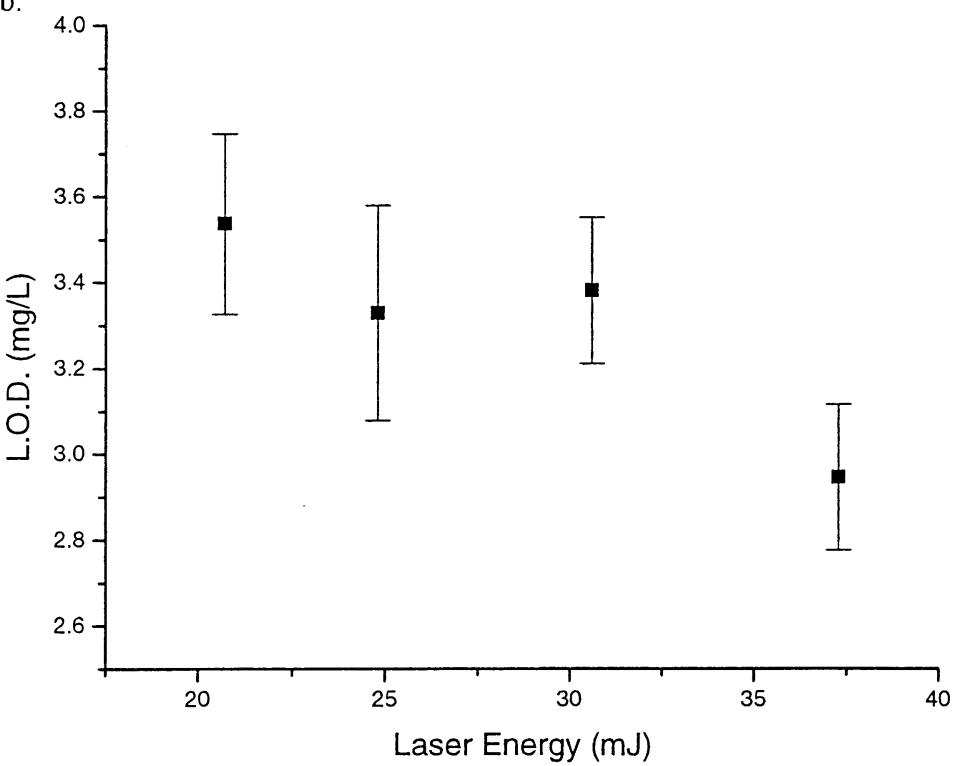

Fig. 7. Laser energy dependence of (a) calibration sensitivity for the K-sample solutions, and (b) limit of detection. Calibration sensitivity is defined as the slope in the calibration curve of concentration. The laser pulse is at $355 \mathrm{~nm}$.

the time-resolved LIB spectrum were not optimized. The gate width and delay time were simply based on the parameters optimized in the $\mathrm{Na}$ analysis.

As the laser at different wavelengths of 266 ,
355 , and $532 \mathrm{~nm}$ is applied with the energy fixed at approximately $13 \mathrm{~mJ}$, the slopes of the correlation plots for $\mathrm{K}$ appear to increase with a decrease of the laser wavelength (Fig. 8). Thus the corresponding detection limits are determined to 


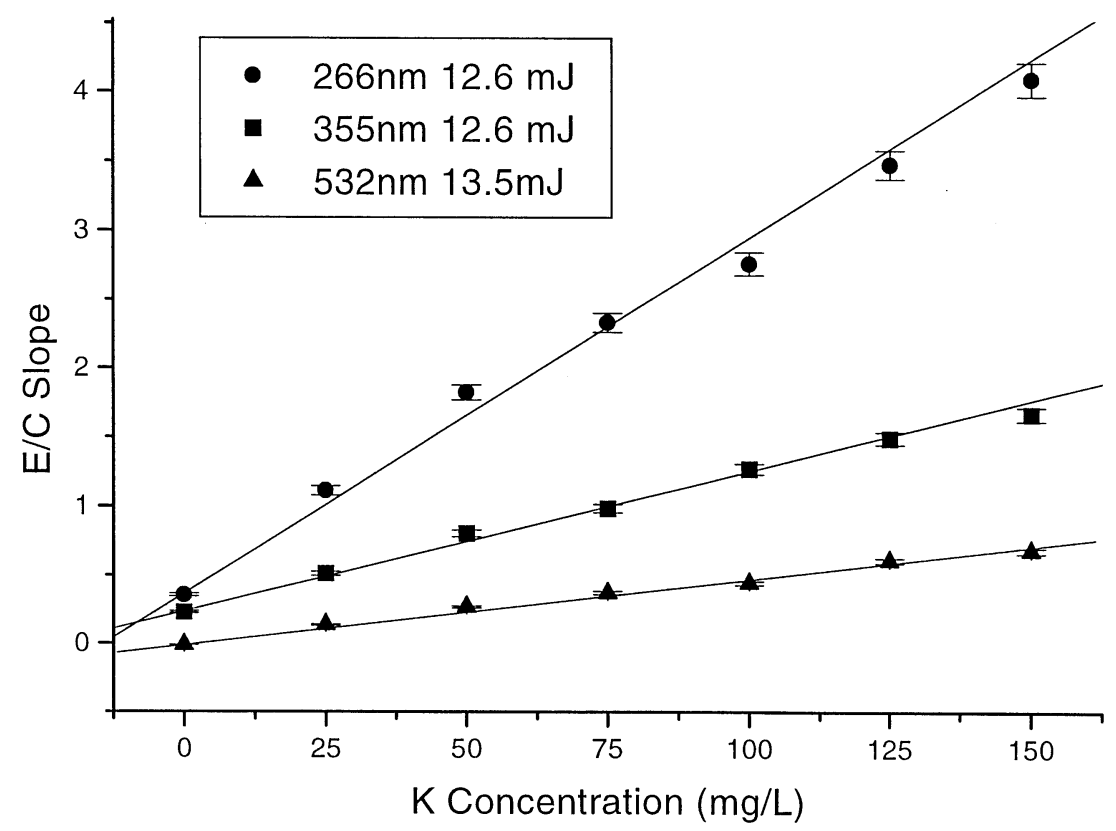

Fig. 8. Calibration curves of concentrations for the $\mathrm{K}$ sample at different pulse wavelengths and energies. (a) Laser pulse at $266 \mathrm{~nm}$ and $12.6 \mathrm{~mJ}, \mathrm{LOD}=1.1 \mathrm{mg} / \mathrm{l}$; (b) $355 \mathrm{~nm}$ and $12.6 \mathrm{~mJ}, \mathrm{LOD}=2.5 \mathrm{mg} / \mathrm{l}$; and (c) $532 \mathrm{~nm}$ and $13.5 \mathrm{~mJ}, \mathrm{LOD}=4.1 \mathrm{mg} / 1$.

be $1.1 \pm 0.2,2.5 \pm 1.0,4.1 \pm 1.5 \mathrm{mg} / 1$ for the 266 , 355 and $532 \mathrm{~nm}$, respectively.

\section{Conclusion}

We have applied the single-shot time-resolved LIB technique to the elemental analysis in the liquid droplets. An electrospay ionization device is employed to generate the microdroplets. The obtained LIB emission signals fluctuate seriously, but such scattered data are advantageous to set up a correlation plot.

By taking advantage of the biased voltage on the electrospray ionization needle, we have collected the produced electrons upon laser-irradiation of the droplets. The resulting current linearly correlates with the corresponding LIB emission of the droplet on single breakdown events. The slope thus obtained is indicative of the sample concentration and can be used to construct a calibration curve for the determination of the analytical sensitivity and the limit of detection. In this manner, we have demonstrated the elemental analysis of $\mathrm{Na}, \mathrm{K}$ and $\mathrm{Al}$. The detection limits tend to be improved by applying a laser with larger pulse energy but shorter wavelengths.

The microdroplet generation facilitates the sample evaporation, atomization, excitation, and ionization efficiency in LIBS. Therefore, the pulse energy used for the sample breakdown is greatly reduced, such that the radiation sources may be extendible to tunable lasers with weak output energies. It should be worthwhile to apply a laser radiation with resonance wavelength to the elemental analysis, particularly for the detection of trace metal binding in biochemical compounds. In comparison with other correlation methods, our presentation in this work is more straightforward to yield concentration information and finds potential applications for biomolecules.

\section{Acknowledgements}

This work is supported by the National Science Council and Chinese Petroleum Company of the 
Republic of China under Contract No. NSC892119-M-002-007.

\section{References}

[1] K. Laqua, N. Omenetto, Analytical Laser Spectroscopy, Wiley, New York, 1979, pp. 47-118.

[2] L.J. Radziemski, T.R. Loree, D.A. Cremers, N.M. Hoffman, Time-resolved laser-induced breakdown spectrometry of aerosols, Anal. Chem. 55 (1983) 1246-1252.

[3] L.J. Radziemski, D.A. Cremers, in: L.J. Radziemski, D.A. Cremers (Eds.), Laser-induced Plasma and Applications, Marcel Dekker, New York, 1989.

[4] H. Schroder, I. Schechter, R. Wisbrun, R. Niessner, in: L.D. Laude (Ed.), Excimer Lasers: The Tools, Fundamentals of their Interactions with Matter, Fields of Applications, Kluwer Academic Publishers, Dordrecht, The Netherlands, 1994, pp. 269-287.

[5] Y.-I. Lee, K. Song, J. Sneddon, in: J. Snedden, T.L. Thiem, Y.-I. Lee (Eds.), Lasers in Analytical Atomic Spectroscopy, VCH, New York, 1997, pp. 197-235.

[6] C.M. Davies, H.H. Telle, D.J. Montgomery, R.E. Corbett, Quantitative analysis using remote laser-induced breakdown spectroscopy (LIBS), Spectrochim. Acta Part B 50 (1995) 1059-1075.

[7] N.H. Cheung, E.S. Yeung, Single-shot elemental analysis of liquids based on laser vaporization at fluences below breakdown, Appl. Spectrosc. 47 (1993) 882-886.

[8] S. Nakamura, Y. Ito, K. Sone, Determination of an iron suspension in water by laser-induced breakdown spectroscopy with two sequential laser pulses, Anal. Chem. 68 (1996) 2981-2986.

[9] L. Xu, V. Bulatov, V.V. Gridin, I. Schechter, Absolute analysis of particulate materials by laser-induced breakdown spectroscopy, Anal. Chem. 69 (1997) 2103-2108.

[10] P. Fichet, P. Mauchien, C. Moulin, Determination of impurities in uranium and plutonium dioxides by laserinduced breakdown spectroscopy, Appl. Spectrosc. 53 (1999) 1111-1117.

[11] D.A. Cremers, L.J. Radziemski, T.R. Loree, Spectrochemical analysis of liquids using the laser spark, Appl. Spectrosc. 38 (1984) 721-729.

[12] J.R. Wachter, D.A. Cremers, Determination of uranium in solution using laser-induced breakdown spectroscopy, Appl. Spectrosc. 41 (1987) 1042-1048.

[13] H.A. Archontaki, S.R. Crouch, Evaluation of an isolated droplet sample introduction system for laser-induced breakdown spectroscopy, Appl. Spectrosc. 42 (1988) 741-746.
[14] R. Knopp, F.J. Scherbaum, J.I. Kim, Laser-induced breakdown spectroscopy (LIBS) as an analytical tool for the detection of metal ions in aqueous solutions, Fresenius J. Anal. Chem. 355 (1996) 16-20.

[15] L.M. Berman, P.J. Wolf, Laser-induced breakdown spectroscopy of liquids: aqueous solutions of nickel and chlorinated hydrocarbons, Appl. Spectrosc. 52 (1998) $438-443$.

[16] Y. Oki, K. Furukawa, M. Maeda, Extremely sensitive Na detection in pure water by laser ablation atomic fluorescence spectroscopy, Opt. Commun. 133 (1997) 123-128.

[17] W.F. Ho, C.W. Ng, N.H. Cheung, Spectrochemical analysis of liquids using laser-induced plasma emissions: effects of laser wavelength, Appl. Spectrosc. 51 (1997) 87-91.

[18] C. Aragon, J.A. Aguilera, F. Penalba, Improvements in quantitative analysis of steel composition by laser-induced breakdown spectroscopy at atmospheric pressure using an infrared Nd:YAG laser, Appl. Spectrosc. 53 (1999) 1259-1267.

[19] D.A. Cremers, J.E. Barefield II, A.C. Koskelo, Remote elemental analysis by laser-induced breakdown spectroscopy using a fiber-optic cable, Appl. Spectrosc. 49 (1995) 857-860.

[20] I.B. Gornushkin, B.W. Smith, G.E. Potts, N. Omenetto, J.D. Winefordner, Some considerations on the correlation between signal and background in laser-induced breakdown spectroscopy using single-shot analysis, Anal. Chem. 71 (1999) 5447-5449.

[21] M.G. Ikonomou, A.T. Blades, P. Kebarle, Electrosprayion spray: a comparison of mechanisms and performance, Anal. Chem. 63 (199 1) (1989) 1998.

[22] G.E. Agnes, G. Horlick, Electrospray mass spectrometry as a technique for elemental analysis: preliminary results, Appl. Spectrosc. 46 (1992) 401-406.

[23] G.C. Turk, Imaging the active flame volume for pulsed laser-enhanced ionization spectroscopy, Anal. Chem. 64 (1992) 1836-1839.

[24] C.B. Ke, K.C. Lin, Laser-enhanced ionization detection of $\mathrm{Pb}$ in seawater by flow injection analysis with on-line preconcentration and separation, Anal. Chem. 71 (1999) 1561-1567.

[25] N. Omenetto, Th. Berthoud, P. Cavalli, G. Rossi, Analytical laser-enhanced ionization studies of thallium in the air-acetylene flame, Anal. Chem. 57 (1985) 1256-1261.

[26] K.D. Su, K.C. Lin, W.T. Luh, Application of laser-enhanced ionization: atomization efficiency determination, Appl. Spectrosc. 46 (1992) 1370-1375. 